ARTÍCULOS DE INVESTIGACIÓN

\title{
Hacia un currículo ambientalmente sustentable para las carreras de Derecho
}

\author{
Towards an environmentally sustainable curriculum for Law careers
}

\author{
Mónica Arnouil Seguel \\ Universidad de La Frontera, Chile
}

\begin{abstract}
RESUMEN Los currículos universitarios de pregrado en Derecho presentan un bajo nivel de inclusión ambiental, tema preocupante en un contexto educativo formador, por cuanto son precisamente los instrumentos de gestión educativa a través de los cuales se logra definir e integrar el contenido ético inmerso en la axiología jurídica y traspasarlo a las aulas en una perspectiva de formación profesional. Desde un modelo educativo de adscripción neoliberal, Chile ha delineado la concepción curricular hacia la eficacia pedagógica, enfoque que lo ha visibilizado como un país con alta oferta para la educación superior universitaria, pero no necesariamente en proporcionar al estudiante los conocimientos necesarios para su futuro laboral. Todo esto resulta paradójico en un país con alta tasa de conflictualidad ambiental y un legado político-social de privación de derechos, que, a lo menos, debe refocalizar las políticas públicas hacia un discernimiento ético-educativo, garante de los principios axiológicos de la acción educativa.
\end{abstract}

PALABRAS CLAVE Derecho ambiental, educación, universidad, currículo, desarrollo sustentable.

ABSTRACT Law curricula have a low level of environmental inclusion. This is highly worrying in a formative educational context, because they're precisely the instruments of educational management through which it's possible to define and integrate the ethical content that is immersed in the legal axiology and transfer it to the classroom in a professional training perspective. From an educational model of neoliberal ascription, Chile has outlined the curricular conception towards pedagogical effectiveness. This approach has determined that it is seen as a country with a high educational offer for university higher education, but not necessarily in providing the student with the knowledge necessary for their future work. All of this is paradoxical in a country with a high rate of environmental conflict and a political and social legacy of deprivation of rights, which at least should have refocused public policies towards an ethical-educational discernment, guarantee of the principles of educational action. 
KEYWORDS Environmental law, education, university, curriculum, sustainable development.

\section{Introducción}

A través del compromiso ambiental que asumieron los Estados en la década de los ochenta como resultado del trabajo de la Comisión Mundial de Medio Ambiente y Desarrollo de Naciones Unidas - redefinido en el Principio 3 de la Declaración de Río de 1992-, se logró consensuar a nivel regional la necesidad de lograr con urgencia un nuevo tipo de desarrollo que asegure el bienestar económico de las generaciones actuales y futuras protegiendo al mismo tiempo los recursos ambientales de los cuales dependen (Arroyave-Cabrera y Miller, 2017).

Bajo esta nueva forma de comprender la sociedad desde lo ambiental, se logró intensificar la integración de los países de la región hacia estándares de economía globalizada (Pérez Sola, 2017) y se planificó un lento y paulatino diseño políticojurídico que busca obtener medidas ambientales tanto económica como socialmente apropiadas a las necesidades y a las problemáticas sentidas por las comunidades.

Visto desde la perspectiva de desarrollo, se instaló la necesidad de generar un marco ético-normativo común que conduzca a alianzas entre las generaciones presentes y futuras para asegurar condiciones dignas de vida, esto es, acordes a la naturaleza humana en una comunidad indivisible entre la sustentabilidad social, basada en el principio de equidad, la sustentabilidad ambiental (Saura Calixto y Hernández Prados, 2008; Cortés-Peña, 2016), que requiere que las funciones útiles de los ecosistemas permanezcan en el tiempo, y la económica, fundada en que todo lo anterior sea posible y rentable (Fernández Niklitschek, 2013).

Desde el paradigma de sustentabilidad ambiental, los Estados se han abocado a priorizar aquellas políticas públicas que busquen promover el fortalecimiento armónico entre medioambiente, pobreza y desarrollo económico, en una tarea que desde las ciencias jurídicas significa repensar y contribuir al surgimiento de un nuevo conocimiento jurídico que permita impulsar, a su vez, novedosos avances en el campo del derecho positivo, como señalara Brañes (2006), que solo son posibles cuando están apoyados por progresos en el pensamiento jurídico.

Las universidades, como centros de aprendizaje y de formación, ostentan un rol ético fundamental en este nuevo conocimiento jurídico ambiental, pues son, finalmente, las que diseñan curricularmente la agenda formativa de los futuros líderes que deberán decidir el patrimonio ambiental de todos (Sandoval Escobar, 2012). En este contexto, las escuelas de Derecho se circunscriben a un deber de facilitar este compromiso ético e integrar los principios del desarrollo sustentable a los objetivos curriculares, esto es, generar currículos de formación que promuevan una real y oportuna reflexión acerca del valor de la naturaleza y la protección de sus recursos. 


\section{Una mirada a la génesis del currículo universitario}

Para comprender la génesis del currículo universitario en Chile, necesariamente se debe revisar en forma crítica el sentido que tuvo su creación dentro del gran proyecto de modernización del país y que surge a partir de un proyecto político autoritario que apuntó a refundarnos culturalmente (Prieto Bafalluy, 1983).

Revisar este modelo educativo desde una mirada integral para avanzar en equidad es una meta pendiente, ya que no basta con disminuir brechas económicas entre los grupos sociales, sino que es necesario gestar un cambio de paradigma en la forma de comprender el sentido de la educación como variable de crecimiento de los pueblos y como un elemento fundamental de la respuesta de innovación del país, fuente de creación de oportunidades que contribuyan al desarrollo económico y social (Ugarte, 2013).

Para impulsar el desarrollo de una nueva forma de comprender el compromiso ético de la universidad como pilar de desarrollo de los pueblos, se debe informar, además, acerca de la forma en la cual compromete su responsabilidad social. Así, nos adentramos a la ardua tarea que enfrenta la universidad para responder a su mandato como gestor educativo y a analizar su principal instrumento de gestión, el currículo.

En una perspectiva de oferta-demanda, nos enfocamos en un currículo de formación que solo debería mostrar una oferta a un cliente que desea adquirirlo; por un lado, esta visión aparece demasiado simplista para conformar por sí misma las bases conceptuales de un proyecto educativo universitario y, por otra, desconoce las muchas y complejas interacciones que se producen o deberían producirse en la definición de un currículo de formación profesional.

La experiencia demuestra que en su diseño intervienen consideraciones relativas a las demandas y preferencias que se plantean a las profesiones, a las tradiciones disciplinarias, a las condiciones impuestas por las organizaciones gremiales y, además, a los estilos de la institución formadora; por tanto, pareciera ser que no es puramente una actividad racional-técnica, sino también una acción propia de la política pública, esto es, trasciende al ámbito de responsabilidad exclusiva de las universidades. La falta de cultura en el ámbito de la planificación docente en nuestras universidades contrasta con el exceso de cultura en el campo de la estructura y de la reforma de los planes de estudio, pues hay más interés por lograr un mayor nivel de exigencia en relación con la calidad de la formación universitaria, desde una perspectiva más pedagógica y universitaria y menos formal e interesada (Martínez Martín, Buxarrais Estrada y Esteban Bara, 2002).

Esta primera distinción en el análisis no puede desprenderse de la génesis y evolución que el propio modelo económico chileno ha impreso durante las últimas décadas al sector educacional, y refleja la concepción educativa que se relaciona en forma directa con la política educacional, que finalmente tributa a un modelo de visión de sociedad y de mundo. 
Detenerse a analizar este último punto parece destacable, por cuanto aparece implícita en ello una obligación de responder, por parte del aparato estatal, a las necesidades y expectativas colectivas de sus ciudadanos en torno a lo educativo, esto es, considerar y orientar el currículo formativo desde las propias valoraciones que los usuarios hacen del fenómeno educativo, desde su propia cultura y desarrollo. Dicho de otra forma, a través del diseño curricular como instrumento de política educativa, el Estado debe otorgar a sus representados la opción de considerar sus expectativas individuales y colectivas, para dar pertinencia y fundamento al mandato educativo. Como señalan Bolaños y Molina Bogantes (2007), para lograr un real mejoramiento de la educación debe existir la máxima pertinencia posible entre la eficacia pedagógica y el entorno social, cultural y económico de un pueblo, pues ningún análisis de la evolución del currículo en Chile puede prescindir de la reflexión acerca de los cambios sociopolíticos que ha experimentado el país en los últimos cuarenta años. Como antecedente a lo señalado, se debe acordar que a nivel de política educativa y curricular desde los años setenta, Chile ha vivenciado tres etapas marcadas: la primera, liderada por el Régimen Militar de la época y asociada a procesos de coerción, represión y ruptura (1973-1979); la segunda, enmarcada en la flexibilidad curricular y un currículo centrado en la persona (1980-1989); y una tercera caracterizada por la creación e implementación de la Ley Orgánica Constitucional de Educación (LOCE), que sostuvo las proyecciones curriculares del Régimen Militar (Cabaluz Ducasse, 2015).

Se puede advertir que en el primer periodo se diseñó una política educacional de marcada visión privatista, en que la educación pierde su condición de derecho fundamental y social y comienza a concebirse como un bien de consumo y un instrumento de lucro. Para el segundo periodo, la educación se reorientó desde estándares de calidad hacia un concepto de apropiabilidad, por lo que se delegó la facultad de su resolución a la esfera de la autonomía del mercado, casi sin intervención estatal (Gentili y Suárez, 2004) y, de esta forma, se incorporaron instrumentos de gestión financiera asociados a un mercado de competitividad.

Ya nos advertían Gustavo Hawes y Karenina Troncoso:

Los problemas trascendentes de la sociedad actual y futura, los desafíos que plantean y la necesidad de formar personas para contribuir a ella requieren competencias en nuestros profesionales que en la actualidad no estamos contribuyendo a formar plenamente. Se hace cada vez más evidente la fragilidad e insuficiencia de una formación profesional centrada exclusivamente en el conocimiento de contenidos y competencias especializadas. La Universidad, después de haber enfrentado los retos más urgentes de infraestructura, perfeccionamiento e investigación, considera de importancia estratégica abordar el rediseño y validación de los currículos de formación de pregrado. El proyecto producirá nuevos currículos de las carreras profesionales, así como las transformaciones organizacionales y de infraestructura apro- 
piadas, dejando instaladas a nivel institucional competencias y habilidades que la empoderen en el cumplimiento de su misión (Hawes Barrios y Troncoso, 2007: 89).

¿Qué ocurrió a nivel curricular en estos periodos? Se pudo constatar que tradujeron la evolución de una política educativa que, desde un diseño curricular de carácter rígido, avanzó hacia una mayor flexibilidad y se centró en el esfuerzo individual y la evaluación.

Finalmente, en un tercer periodo, se observa, a nivel curricular, una etapa fuertemente marcada por el establecimiento de la Constitución Política de 1980 y la dictación de la Ley Orgánica Constitucional de Educación, que prioriza en sus lineamientos generales la libertad de enseñanza y garantiza la libre elección de los padres para escoger el establecimiento de sus hijos. Se trata de un periodo en el que se otorga al Estado un rol garante de estos derechos, donde el diseño de los lineamientos curriculares estuvo adscrito al modelo económico imperante, que priorizó la aprobación de objetivos fundamentales y contenidos mínimos obligatorios para la educación básica y media, a través de la creación del Consejo Superior de Educación (Oliva Ureta, 2010).

¿Qué ocurre hoy? En Chile existe una alta oferta de educación superior con 60 universidades, 27 de las cuales pertenecen al Consejo de Rectores de las Universidades Chilenas y 33 cuentan con dependencia privada, cada una de ellas con autonomía para estructurar su propio proyecto educativo que, a su vez, está orientado por una política ministerial en un Chile centrado en el mercado como capital educativo.

A nivel de propuesta, el diseño curricular ha obedecido hasta hoy a un modelo político-económico de planificación que integra a las diferentes instancias de formación educacional: un currículo que identifica las competencias laborales y permite establecer las funciones y tareas que implica el desempeño profesional a partir de identificar los conocimientos, habilidades y aptitudes que se requieren para lograr dicho desempeño, los cuales finalmente son relevados en forma de comportamientos evaluables. Dicho de otra forma:

El enfoque basado en competencias aplicado correctamente se caracteriza por utilizar recursos que simulan la vida real o reflejan situaciones de trabajo real y experiencias de trabajo; ofrecer una gran variedad de recursos para que los estudiantes analicen y resuelvan problemas; enfatizar el trabajo cooperativo y colaborativo apoyado por el docente; identificar cuidadosamente las competencias que los estudiantes desarrollarán, verificándolas en sus trayectorias; orientar la enseñanza hacia el desarrollo de cada competencia con su respectiva evaluación; considerar en el diseño de las evaluaciones de aprendizaje tanto los recursos cognitivos, procedimentales, actitudinales, como también el desempeño de la competencia como principal fuente de evidencia; tomar en cuenta el ritmo de los alumnos para verificar su progreso en el programa según las competencias demostradas; brindar retroalimentación sis- 
temática y permanente a fin de orientar las experiencias de aprendizaje; planificar cuidadosamente el programa en su totalidad y aplicar una evaluación sistemática para mejorarlo (Moyano Díaz, Vásquez y Faúndez, 2012: 8).

No obstante, la amplia oferta educativa no pareciera estar mayormente comprometida con la formación universitaria, pues se cuenta con universidades que no necesariamente proporcionan al estudiante el conjunto de conocimientos requeridos para su futuro ejercicio profesional, por lo menos bajo un modelo de sociedad con conocimientos actualizados y aplicables en contextos de aprendizaje, y desde la resignificación y comprensión valórica de sus metas, es decir, una formación obligada con la seriedad, el rigor, la crítica y la revisión permanente, de forma de situar a la educación como un mecanismo de protección de los valores intelectuales y éticos que la integran. Esto, básicamente, porque su mandato ético no puede abordarse sólo desde una perspectiva declarativa, sino que obliga a una seria y permanente revisión de todos los programas y acciones desarrollados al interior de sus aulas.

Si se analiza la aplicabilidad de este modelo curricular desde el área ambiental, se distinguen distintos abordajes en las universidades: mientras unas desarrollan contenidos específicos en sus mallas, otras solo declaran recomendaciones o expectativas ambientales, y las menos tributan a un cambio de malla curricular, de manera de incorporar asignaturas de formación general sobre medioambiente, circunscritas a valores y temáticas propias de la profesión, desde la perspectiva de la sustentabilidad, visión con la cual las bases conceptuales de la educación chilena parecieran no coincidir.

Existen categorías de intervención del tema ambiental (Barreto y Neme Chaves, 2014) en las universidades chilenas. La más básica, una primera aproximación del alumno al análisis de su ambiente, es a través de seminarios y charlas específicas sobre el tema, lo que implica llevar personajes destacados en los ámbitos relacionados con el ejercicio profesional para que presenten cómo influye el compromiso medioambiental en su profesión. Una segunda categoría, que tampoco implica cambio de malla curricular, estaría dada por solicitar a cada uno de los profesores que aborde los contenidos de sus programas desde la óptica de los desafíos medioambientales relacionados con cada uno de los temas que se tratan en el curso. La tercera categoría, que se desprende del análisis del autor, señala que, antes del cambio de malla curricular, se cambien los contenidos de los programas de manera que se asuman explícitamente en relación al desarrollo sustentable. Por último, una cuarta categoría o estadio superior es un currículo enfocado al desarrollo sustentable, lo que implicaría, necesariamente, la reelaboración de la malla curricular de manera de incorporar asignaturas de formación general sobre desarrollo sustentable que formen al estudiante en valores y asignaturas específicas, y que aborden cada una de las temáticas propias de la profesión desde la perspectiva del desarrollo sustentable. Esto significa 
lograr que, a través de sus asignaturas, los estudiantes sean partícipes de prácticas sustentables, lo cual requiere una docencia para el desarrollo de esta temática, junto con la participación activa de los jóvenes en charlas y seminarios que traten aspectos medioambientales de sus profesiones (Mercado, 2010).

La visión de sustentabilidad ambiental al interior de las aulas de Derecho (Herrera-Mendoza y otros, 2016) debiera, como señala el paradigma de la complejidad, permear el diseño curricular por competencias que rigen hoy en el sistema educativo formal e impulsar a la institución educativa a lograr un liderazgo y trabajo en equipo que priorice la calidad del aprendizaje compartido por toda la comunidad educativa, con estrategias de impacto que promuevan la formación integral de los estudiantes, de forma que interioricen y vinculen un proyecto ético de vida con un claro compromiso frente a las grandes crisis de la sociedad, con ímpetu investigador y calidad profesional.

En este contexto, uno de los principales retos de la educación está en superar las limitaciones disciplinares a nivel científico tradicional y ofrecer soluciones eficaces a la grave crisis ambiental (Ibáñez, Amador Muñoz y Mateos Claros, 2017).

\section{El derecho y su rol axiológico en la educación}

Con todo, se debe consensuar que referir a la temática ambiental implica un cambio en la lógica jurídica clásica y en la epistemología del derecho en general. Se ha debido comprender la necesidad de remirar y revisar el derecho como sistema regulador de la conducta humana desde el impacto y la crisis ambiental.

Ahora bien, para alcanzar una epistemología jurídica que visualice el derecho como eje de construcción y transformación social o que realmente merezca reconocerse como tal, debieran producirse a lo menos tres cambios importantes en nuestra percepción del derecho y de la sociedad (Teubner, 2002).

Autores clásicos como Hegel han señalado que la ética es el concepto de la libertad convertido en mundo existente y naturaleza de la conciencia de sí misma. Desde esta perspectiva, la libertad se asume como un derecho a la particularidad de los individuos, que es el modo aparente de existencia del ethos. Hegel nos recuerda en Filosofía del derecho la pregunta de un padre a un pitagórico acerca del mejor modo de educar éticamente al propio hijo, cuya respuesta fue: «Que tú lo hagas un ciudadano de un Estado con leyes justas». De esta forma, podemos identificar «deber»y «derecho», esto es, que el hombre con la ayuda de lo ético tiene derechos en cuanto tiene deberes $\mathrm{y}$ a su vez tiene deberes en cuanto tiene derechos.

El primero, pasar del realismo al constructivismo; el segundo, avanzar desde la construcción individual de la realidad a la construcción social; y el tercero, transmutar desde la concepción del derecho como un sistema de normas aplicable a la sociedad que regula, a un derecho como sujeto epistémico. Todos estos cambios nos 
invitan a repensar las formas tradicionales de concepción del derecho, pues llevan a una cierta modificación de las posiciones kantianas, y abren un nuevo camino en la teoría jurídica y social.

Sea cual sea la revisión que se haga desde la epistemología, se debe reconocer que el derecho ha impulsado la integración de una visión regional, con una estructura normativa específica que no solo busca normar las actividades humanas en la protección de los derechos ambientales, sino también desentrañar sus bases axiológicas para determinar su protección.

Brañes (2006) se refiere al constitucionalismo verde como un fenómeno jurídico de las décadas de los setenta y ochenta en América Latina, que se plasmó en la integración de los derechos que regulan la relación entre humanos y la naturaleza al más alto nivel jurídico del derecho interno de los Estados, de forma que se comprendió la necesidad de elevar la protección del ambiente a la categoría de derecho fundamental.

En esta ardua tarea de promoción de derechos, se plantea la pregunta: ¿dónde se ubica la universidad como eje gestor del desarrollo de los pueblos? Esta interrogante surge como una reflexión esencial en el discernimiento ético-educativo respecto del real compromiso que la universidad está dispuesta a asumir para avanzar en metas de sustentabilidad. No basta con generar sistemas de protección legales si no se es capaz de impulsar la necesidad de transformar los esquemas de pensamientos arraigados que, como señaló Morin (1999), no han contribuido a construir ni invitan a imaginar una educación para vivir mejor, con mayor calidad de vida.

Se puede coincidir en que es urgente transformar el pensamiento para crear conocimiento ambientalmente sustentable y tomar decisiones conscientes, consistentes y compatibles con los paradigmas ambientales que nos movilizan en nuestra relación humanos-naturaleza, lo cual requiere una revisión urgente de los diseños curriculares al interior de las escuelas de Derecho.

Uno de los principales retos está en planificar currículos que superen las limitaciones disciplinares a nivel científico tradicional y ofrezcan al estudiante la oportunidad de vivenciar soluciones eficaces a la grave crisis ambiental mundial, por cuanto la tarea urgente no está en medir competencias para el mercado, sino en obtener profesionales competentes para enfrentar los problemas que hoy debe resolver la sociedad. Dicho de otra forma, es tarea urgente que las universidades desarrollen una enseñanza explícita en valores, ya que solo una educación ética formal se encamina al desarrollo armónico e integral de las personas y, por tanto, es un elemento esencial para la sobrevivencia de la sociedad en su conjunto.

Para ello se propone integrar los principios del derecho ambiental al gobierno transversal de las mallas curriculares, desde una perspectiva biocéntrica, que integre resultados de aprendizaje que orienten a la protección y la conservación de derechos humanos ambientales. Parece primordial focalizar una docencia encaminada a formar profesionales capaces de contribuir al desarrollo sustentable, lo cual obliga a in- 
cluir en las mallas curriculares y en las distintas asignaturas el tema medioambiental a partir de la perspectiva de cada una de las carreras, una formación general valórica que asegure el compromiso de los futuros tomadores de decisión con acciones que no atenten contra la sustentabilidad y permitan implantar un sello distintivo a los estudiantes de una casa de estudios (Mercado, 2010). Sin embargo, nos encontramos en una sociedad que sostiene una crisis de sus bases éticas (Hortal, 1994) y por consiguiente, como señala Mercado, un desafío de las universidades de hoy está en ajustar una planificación curricular hacia una educación en valores, por cuanto el acceso a la justicia ambiental demanda en forma progresiva una preparación especial de abogados y jueces, que difícilmente es proporcionada por la enseñanza que hoy reciben sus estudiantes.

Como Brañes sostenía, es preciso una educación en, sobre y para el ambiente. Dicho de otro modo, se requiere mejorar la relación del ser humano con su entorno natural a través de medios coercitivos, en este caso del derecho ambiental. Lo anterior implica y exige al mismo tiempo que la universidad que quiera plantearse a sí misma como socialmente responsable debe transitar por un cuestionamiento epistemológico de lo que enseña (Pol y Castrechini, 2013; Ortega Ruiz y Romero Sánchez, 2009), de la visión del mundo que promueve en sus aulas y del tipo de ciencia que ofrece a los estudiantes. Para qué enseñamos lo que estamos enseñando y cuál es la trascendencia de los conocimientos que estamos dispuestos a dar a los futuros decisores del patrimonio ambiental debieran ser los principales y continuos cuestionamientos de un currículo que promueva el sentido crítico dentro del contexto universitario.

\section{Conclusiones}

El diseño curricular y su aplicación en el pregrado universitario en Chile se encuentran fuertemente afianzados por la visión de mundo y sociedad que hemos construido. De la misma forma, la mayor o menor vinculación con las competencias profesionales o la empleabilidad laboral condicionan la perspectiva de sociedad que queremos para nuestros próximos años.

Chile sostiene un modelo neoliberal de desarrollo económico desde los años setenta que nos afianza hacia un vértice de economía capitalista, el cual, analizado desde la esfera de política educacional y ambiental, delimita las formas y los usos que hacemos del ambiente que nos rodea; también a nivel de aplicabilidad normativa, debilita la creatividad y la libertad del conocimiento, al restringir sus líneas programáticas hacia la enseñanza como un requisito de contenido y no hacia el aprendizaje como una meta social.

La sustentabilidad como forma de comprender la relación entre humanos y naturaleza (Amérigo Cuervo-Arango, García y Sánchez Núñez, 2013; Pereira Nocera y otros, 2018) se ajusta a una forma solidaria de comprensión de sociedad en que lo 
ambiental es un componente esencial para decidir el uso de nuestros recursos naturales. Chile, adscrito a la meta de promover la sustentabilidad, apuesta a un cambio en la lógica jurídica que posibilite regular en forma colaborativa los graves conflictos que surgen en esta desigual relación.

Hoy, por tanto, es necesario traspasar las esferas tradicionales de las ciencias jurídicas para aproximarnos a una regulación que se ajuste a resolver los conflictos reales que surgen en el uso y aprovechamiento de los recursos naturales, todo lo cual debe hacerse desde la comprensión del rol de la universidad como pilar ético en la formación de las futuras generaciones, ya que es precisamente en las escuelas de Derecho donde se forma a los futuros decisores del destino del patrimonio ambiental, dominio legítimo de todos los chilenos.

Desde esta perspectiva, se identifica con urgencia que los currículos de pregrado universitarios sean también replanificados y conformados desde una mirada integral de responsabilidad social y ética como capital ambiental (Corral-Verdugo y otros, 2017; Cruz Rodríguez, 2014; Tovar-Gálvez, 2017; Vega y otros, 2009; Vílchez, 2009) y, además, sean capaces de traducir solidariamente concepciones de justicia ambiental (Munévar Quintero, 2013), es decir, el cómo ajustarlos a una demanda por acceso igualitario en pro de la defensa de los derechos ambientales ciudadanos. Solo haciendo reflexiones de este orden se puede integrar una visión transformadora de mundo a los futuros líderes que se forman en el claustro universitario y con ello cumplir con la obligación de responder al mandato ético que la sociedad entrega a la universidad, el de contribuir a transformarla para mejorarla.

Hoy, currículos lineales, asignaturistas y medidores de parámetros cuantitativos con evaluaciones de contenidos en las ciencias jurídicas tradicionales segmentan y desconfiguran la visión social que debe tener el estudiante frente a su realidad y su entorno, por cuanto se demanda en forma urgente que éste intervenga, participe, investigue y decida frente a las opciones que se le van a presentar en situaciones de crisis (González Jaramillo y Ortiz García, 2011).

En los parámetros actuales se están midiendo competencias, no valores, como formas de comprender el funcionamiento de la sociedad y, por tanto, demanda de los planificadores curriculares una integración de métodos de aprendizaje que reflejen la esencia de los proyectos educativos institucionales, de sus actores, sus recursos y dimensiones. En síntesis, que permitan medir la calidad valórica del aprendizaje que se está obteniendo en el aula, de forma de asegurar que el futuro profesional del derecho decida éticamente frente a los dilemas que va a enfrentar.

\section{Referencias}

Amérigo Cuervo-Arango, María, Juan García y Trinidad Sánchez Núñez (2013). «Actitudes y comportamiento hacia el medio ambiente natural: Salud medioam- 
biental y bienestar emocional». Universitas Psychologica, 12 (3): 845-856. DOI: 10.11144/Javeriana.UPSY12-3.acma.

Arroyave-Cabrera, Jesús Antonio y Toby Miller (2017). «De la ecología de medios a la ecología profunda de medios: Esclarecer la metáfora y visibilizar su impacto medioambiental». Palabra Clave, 20 (1): 239-268. DOI: 10.5294/pacla.2017.20.1.11.

BARreto, Idaly y Samir Ricardo Neme Chaves (2014). «Eficacia de tácticas de influencia en la intención de conducta proambiental». Revista Latinoamericana de Psicología, 46 (2): 111-116. Disponible en http://bit.ly/2HNVejf.

Bolaños, Guillermo y Zaida Molina Bogantes (2007). Introducción al currículo. San José: EUNED.

BRAÑEs, Raúl (2006). La fundación del derecho ambiental en América Latina. Ciudad de México: Programa de las Naciones Unidas para el Medio Ambiente.

Cabaluz Ducasse, Jorge Fabian (2015). «El proyecto curricular de la dictadura cívico-militar en Chile (1973-1990)». Perspectiva Educacional, 54 (2): 165-180. DOI: 10.4151/07189729-Vol.54-Iss.2-Art.340.

Corral-Verdugo, Víctor, Fernanda Inés García-Vázquez, Blanca Fraijo-Sing y César Tapia-Fonllem (2017). «Is it human mind attuned with general ecological principles?». Interamerican Journal of Psychology, 51 (3): 380-397. Disponible en http:// bit.ly/2HOWen3.

Cortés-PeñA, Omar Fernando (2016). «Comportamiento proambiental y desarrollo económico sustentable en jóvenes universitarios». Opción, 32 (9): 387-407. Disponible en http://bit.ly/2HMnClK.

Cruz Rodríguez, Edwin (2014). «Del derecho ambiental a los derechos de la naturaleza: Sobre la necesidad del diálogo intercultural». Jurídicas, 11 (1): 95-116.

Fernández Niklitschek, Pedro (2013). Manual de derecho ambiental chileno. Santiago: Libromar.

Gentili, Pablo y Daniel Suárez (2004). Reforma educacional e luta democrática: Um debate sobre ação sindical docente na América Latina. San Pablo: Cortez.

Hawes Barrios, Gustavo y Karenina Troncoso (2007) «Modelo didáctico universitario». Instituto de Investigación y Desarrollo Educacional. Proyecto Mecesup, TAL-0101. Universidad de Talca.

Herrera-Mendoza, Ketty, Mónica Acuña Rodríguez, María Jesús Ramírez Ordoñez y Marcela de la Hoz Álvarez (2016). «Actitud y conducta pro-ecológica de jóvenes universitarios». Opción, 32 (13): 456-477. Disponible en http://bit.ly/2HMtGeo.

Hortal, Augusto (1994). Ética general de las profesiones. Bilbao: Centros Universitarios de la Compañía de Jesús.

IвÁÑ̃z, Macarena Esteban, Luis Amador Muñoz y Francisco Mateos Claros (2017). «Actitudes del alumnado universitario hacia el medio ambiente: Educación ambiental e innovación». Revista de Humanidades, 31: 17-38. Disponible en http://bit. ly/2LQLWoS. 
GonzÁlez JARAmillo, Suleida y Martha Ortiz García (2011). «Las competencias profesionales en la educación superior». Educación Médica Superior, 25 (3): 234-243.

Martínez Martín, Miquel, María Rosa Buxarrais Estrada y Francisco Esteban Bara (2002). «La universidad como espacio de aprendizaje ético». Revista Iberoamericana de Educación, 29: 17-44. Disponible en http://bit.ly/2LRj4gl.

MoRIN, Edgar (1999). Los siete saberes necesarios para la educación del futuro. Barcelona: Paidós.

Moyano Díaz, Emilio, Marcela Vásquez y Fabiola Faúndez (2012). Cambios en el modelo educativo universitario: Desafíos culturales, curriculares y de gestión. Talca: Universidad de Talca.

Munévar Quintero, Claudia (2013). «La incidencia de la educación jurídica ambiental y la participación en la transformación del conflicto ambiental». Jurídicas, 10 (1): 147-163.

Oliva Ureta, María Angélica (2010). «Política educativa chilena 1965-2009: ¿Qué oculta esa trama?». Revista Brasileira de Educação, 15 (44): 311-328. DOI: 10.159o/ S1413-24782010000200008.

Ortega Ruiz, Pedro y Eduardo Romero Sánchez (2009). «La dimensión ética de la crisis medioambiental: Propuestas pedagógicas». Teoría de la Educación, 21 (1):161-178. Disponible en http://bit.ly/2HOImsX.

Pereira Nocera, Renata, Nayibe Jiménez Rodríguez, Eloy Andrés Herrera Meza, William Velázquez Barrios y Adriana María Estrada Mejía (2018). «Del Convenio Aarhus y sus efectos para el derecho internacional del medio ambiente: Un análisis del pilar de la participación del público». Espacios, 39 (11): 3-13. Disponible en http://bit.ly/2LOjwM9.

PÉrez Sola, Nicolás (2017). «El derecho-deber de protección del medio ambiente». Revista de Derecho Político, 100: 949-986. DOI: 10.5944/rdp.100.2017.20723.

Pol, Enric y Angela Castrechini (2013). «¿Disrupción en la educación para la sostenibilidad?». Revista Latinoamericana de Psicología, 45 (3): 333-347. DOI: 10.14349/ rlp.v45i3.1477.

Prieto Bafalluy, Alfredo (1983). La modernización educacional. Santiago: Ediciones UC.

SANDOVAL Escobar, Marithza (2012). «Comportamiento sustentable y educación ambiental: Una visión desde las prácticas culturales». Revista Latinoamericana de Psicología, 44 (1): 181-196. Disponible en http://bit.ly/2LPHpDb.

Saura Calixto, Pilar y María Ángeles Hernández Prados (2008). «La evolución del concepto de sostenibilidad y su incidencia en la educación ambiental». Teoría de la Educación, 20: 179-204. Disponible en http://bit.ly/2LQuQri.

Teubner, Gunther (2002). «El derecho como sujeto epistémico: Hacia una epistemología constructivista del derecho». Doxa, 25: 533-571. Disponible en http://bit. ly/2HRyyOO. 
Tovar-Gálvez, Julio César (2017). «Pedagogía ambiental y didáctica ambiental: Tendencias en la educación superior». Revista Brasileira de Educação, 22 (69): 519-538.

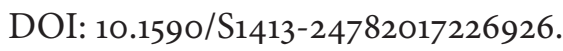

Ugarte, Juan José (2013). Panorama del sistema de educación superior chileno y el aseguramiento de la calidad. Santiago: Ministerio de Educación.

VegA, Pedro, Mario Freitas, Pedro Álvarez y Reinaldo Fleuri (2009). «Educación ambiental e intercultural para la sostenibilidad: Fundamentos y praxis». Utopía y Praxis Latinoamericana, 14 (44): 25-38. Disponible en http://bit.ly/2LNYZHC.

VÍlCheZ, José Eduardo (2009). «La problemática ambiental en los medios: Propuesta de un protocolo de análisis para su uso como recurso didáctico». Enseñanza de las Ciencias, 27 (3): 421-432. Disponible en http://bit.ly/2HLn5QZ.

\section{Sobre la autora}

Mónica Arnouil Seguel es abogada y asistente social. Doctora en Ciencias de la Educación y postdoctoranda en Ciencias de la Educación de la Universidad de La Frontera, Chile. Su correo electrónico es moniarnouil@yahoo.com. 
